\title{
Uncertainty in Electroweak Symmetry Breaking in Models With High Scale Supersymmetry Breaking and its Impact on Interpretations of Searches For Supersymmetric Particles
}

\author{
B. C. Allanach ${ }^{a}$ M A Parker ${ }^{b}$ \\ ${ }^{a}$ Department of Applied Mathematics and Theoretical Physics, Centre for Mathematical Sciences, \\ University of Cambridge, Wilberforce Road, Cambridge CB3 0WA, United Kingdom \\ ${ }^{b}$ Cavendish Laboratory, University of Cambridge, JJ Thomson Avenue, Cambridge CB3 0HE, \\ United Kingdom
}

E-mail: B.C.Allanach@damtp.cam.ac.uk, parker@hep.phy.cam.ac.uk

\begin{abstract}
Some regions of parameter space of the minimal supersymmetric standard model (MSSM) with high scale supersymmetry breaking have extreme sensitivity of electroweak symmetry breaking (EWSB) to the top quark mass through renormalisation group evolution effects. This leads to uncertainties in the predictions which need to be taken into account in the interpretation of searches for supersymmetric particles in these regions. As an example, we provide estimates of the current uncertainties on the position in parameter space of the region which does not break electroweak symmetry in the constrained MSSM (CMSSM). The position of the boundary of EWSB can vary by up to $2 \mathrm{TeV}$ in $m_{0}$ due to the uncertainties coming from the current measurement errors on the top quark mass and from higher order corrections. In this dangerous region, for fixed CMSSM parameters the neutralino lightest supersymmetric particle mass has an associated large uncertainty of order $100 \%$. These uncertainties therefore have a profound effect on the interpretation of LHC supersymmetric particle searches in terms of the CMSSM. We also show how to ameliorate poor convergence of the iterative numerical algorithm that calculates the MSSM spectrum near the boundary of EWSB.
\end{abstract}

Keywords: Supersymmetric Phenomenology, Large Hadron Collider 


\section{Contents}

1 Introduction $\quad 1$

1.1 EWSB in the MSSM 2

2 Uncertainties in Electroweak Symmetry Breaking 5

3 Convergence 12

$\begin{array}{lll}4 & \text { Summary and Conclusions } & 15\end{array}$

\section{Introduction}

Searches for supersymmetric particles are one of the highest priorities for the Large Hadron Collider, and the subject of many recent experimental and theoretical publications. The data taken up until 2011 at a centre of mass energy of $7 \mathrm{TeV}$ has now been extensively analysed. New results have begun to appear from $8 \mathrm{TeV}$ data, and results from the complete $8 \mathrm{TeV}$ data set are expected to appear in 2013. The most stringent limits on the masses of supersymmetric squarks and gluinos come from generic searches using the missing energy signature, with a varying number of high transverse momentum jets $[1,2]$. The searches in Ref. [1] exclude gluino and squark masses below $860 \mathrm{GeV}$ and $1320 \mathrm{GeV}$ respectively in simplified scenarios where only direct decays of these sparticles are allowed and $R$-parity is assumed. In the constrained MSSM (CMSSM) framework [3-7], where the sparticle masses are fixed at a high gauge unification scale $M_{X} \sim 2 \times 10^{16} \mathrm{GeV}$, the searches exclude universal gaugino masses $M_{1 / 2}<640 \mathrm{GeV}$ at low values of universal scalar mass $m_{0}$, and $M_{1 / 2}<$ $300 \mathrm{GeV}$ at high $m_{0}$, approximately independently of the ratio of the two Higgs vacuum expectation values $\tan \beta=v_{2} / v_{1}$ and the trilinear supersymmetry (SUSY) breaking scalar coupling $A_{0}$. In the CMSSM the limits approach the region where electroweak symmetry breaking (EWSB) does not occur, and the search limits are generally not plotted beyond the expected boundary of this region. The same can be seen in the most recent conference interpretations of so far unpublished data from these experiments.

When the LHC runs at higher energy (13 or $14 \mathrm{TeV}$, starting in 2014), the searches will be sensitive to far higher values of $m_{0}$ and $M_{1 / 2}$. It is therefore important to understand the behaviour of the theoretical predictions as the boundary of EWSB is approached. The uncertainty on the location of the boundary needs to be taken into account, as well as the uncertainties on the predicted sparticle masses. These effects can significantly alter the region of validity of the exclusion limits in interpretations of the data in terms of highscale SUSY breaking models (although they of course do not alter the data themselves). In particular, if one is attempting to rule out the CMSSM (i.e. a bona fide top-down analysis), the question of the uncertainty of the position of the electroweak symmetry 
breaking boundary is a physical one: in this case, we must fix the CMSSM parameters and ask if the point is ruled out or not. This is also in principle the case for other top down high-scale models of supersymmetry breaking where the various SUSY breaking terms are set by a small number of parameters. If one is performing a 'simplified model' or 'phenomenological MSSM' approach, one has enough free parameters to make sure that EWSB is broken correctly, and the problem should not arise. In practice, there is much interest from the experiments in ruling out the CMSSM in particular because it is a familiar and a priori reasonable model, and because it serves as a well-studied benchmark for experimental progress in model space.

For a given point in MSSM parameter space, the computer program SOFTSUSY [8] is currently used by ATLAS and CMS to calculate the sparticle spectrum and MSSM couplings, and to determine whether or not the point performs EWSB successfully. We shall therefore also utilise SOFTSUSY to quantify uncertainties due to the position of EWSB. Near the boundary of successful EWSB, SOFTSUSY reports non-convergence of the numerical algorithm, which manifests as a small parameter region on the experimental plots where the interpretation of an experimental search in terms of a high-scale SUSY breaking model cannot be trusted. Refs. [9] and [10] have also recently noticed that at high values of third family scalar masses, sparticle spectrum calculators (including SOFTSUSY) give inaccurate results: the sparticle mass calculation is particularly difficult to solve in this tricky region of parameter space. The region of parameter MSSM space with large scalar masses has received considerable attention in the literature, and is called the focus point, or hyperbolic branch [11-13]. The focus point has received renewed attention recently because if the SUSY breaking scalar trilinear coupling involving Higgs and stops $A_{t}$ is large, then a 125 GeV lightest CP even Higgs boson (as implied by 2012 LHC Higgs search data $[14,15]$ ) may result [16-18].

\subsection{EWSB in the MSSM}

We write the MSSM superpotential in terms of chiral superfields, suppressing gauge indices and neglecting all Yukawa couplings save those of the third family,

$$
W=\mu H_{2} H_{1}+h_{t} Q_{3} H_{2} u_{3}+h_{b} Q_{3} H_{2} d_{3}+h_{\tau} L_{3} H_{1} e_{3},
$$

where the chiral superfields of the MSSM have the following $G_{S M}=S U(3)_{c} \times S U(2)_{L} \times$ $U(1)_{Y}$ quantum numbers

$$
\begin{array}{ll}
L_{i}:\left(1,2,-\frac{1}{2}\right), \quad e_{i}:(1,1,1), \quad Q_{i}:\left(3,2, \frac{1}{6}\right), \quad u_{i}:\left(\overline{3}, 1,-\frac{2}{3}\right), \\
d_{i}:\left(\overline{3}, 1, \frac{1}{3}\right), \quad H_{1}:\left(1,2,-\frac{1}{2}\right), \quad H_{2}:\left(1,2, \frac{1}{2}\right),
\end{array}
$$

and $i \in\{1,2,3\}$ is a family index. We shall write superpartners of SM fields with a tilde.

Minimising the MSSM Higgs potential with respect to the electrically neutral components of the Higgs vacuum expectation values, one obtains the well-known tree-level result for the Higgs mass parameter $\mu$ in the modified dimensional reduction scheme $(\overline{D R})$

$$
\mu^{2}=\frac{\tan 2 \beta}{2}\left[m_{H_{2}}^{2} \tan \beta-m_{H_{1}}^{2} \cot \beta\right]-\frac{M_{Z}^{2}}{2} .
$$


In order to reduce ${ }^{1}$ missing higher order corrections, all quantities in Eq. 1.3 are understood to be evaluated at a $\overline{D R}$ renormalisation scale $Q=M_{S U S Y}$, where $M_{S U S Y}$ is the geometric mean of the two stop masses. $\tan \beta=\left\langle H_{2}^{0}\right\rangle /\left\langle H_{1}^{0}\right\rangle$ is the ratio of the two MSSM Higgs vacuum expectation values and $m_{H_{1,2}}$ are the soft SUSY breaking $\overline{D R}$ mass terms of the Higgs doublets. If $m_{H_{1}}^{2}$ and $m_{H_{2}}^{2}$ and $\tan 2 \beta$ are such that $\mu^{2}>0$ results from Eq. 1.3, the model point may break electroweak symmetry successfully. On the other hand, if $\mu^{2} \leq 0$, electroweak symmetry is not broken successfully and the model point is ruled out. We shall here study current uncertainties in the position in MSSM parameter space of the $\mu=0$ contour that separates the region of successful EWSB from the region of no EWSB. We shall also study the extent to which these matter in terms of searches for supersymmetric particles.

The $m_{H_{i}}^{2}$ terms on the right hand side of Eq. 1.3 are connected to the squark and gluino masses in models of SUSY breaking through the boundary conditions on SUSY breaking terms, imposed at a (typically high) scale $M_{X}$ and through the renormalisation group equations (RGEs), which at one-loop order are [19]

$$
\begin{aligned}
16 \pi^{2} \frac{\partial m_{H_{2}}^{2}}{d t}= & 6\left[\left(m_{H_{2}}^{2}+m_{\tilde{Q}_{3}}^{2}+m_{\tilde{u}_{3}}^{2}+A_{t}^{2}\right) h_{t}^{2}\right]-6 g_{2}^{2} M_{2}^{2}-\frac{6}{5} g_{1}^{2} M_{1}^{2}+\frac{3}{5} g_{1}^{2}\left(m_{H_{2}}^{2}-m_{H_{1}}^{2}+\right. \\
& \left.\operatorname{Tr}\left[m_{\tilde{Q}}^{2}-m_{\tilde{L}}^{2}-2 m_{\tilde{u}}^{2}+m_{\tilde{d}}^{2}+m_{\tilde{e}}^{2}\right]\right) \\
16 \pi^{2} \frac{\partial m_{H_{1}}^{2}}{d t}= & 6\left[\left(m_{H_{1}}^{2}+m_{\tilde{Q}_{3}}^{2}+m_{\tilde{d}_{3}}^{2}+A_{b}^{2}\right) h_{b}^{2}\right]-6 g_{2}^{2} M_{2}^{2}-\frac{6}{5} g_{1}^{2} M_{1}^{2}-\frac{3}{5} g_{1}^{2}\left(m_{H_{2}}^{2}-m_{H_{1}}^{2}+\right. \\
& \left.\operatorname{Tr}\left[m_{\tilde{Q}}^{2}-m_{\tilde{L}}^{2}-2 m_{\tilde{u}}^{2}+m_{\tilde{d}}^{2}+m_{\tilde{e}}^{2}\right]\right)
\end{aligned}
$$

where $t=\ln Q$. When $\left(m_{H_{2}}^{2}+m_{\tilde{Q}_{3}}^{2}+m_{\tilde{u}_{3}}^{2}+A_{t}^{2}\right)$ is large, the renormalisation of $m_{H_{2}}$ (and therefore its value at $\left.M_{S U S Y}\right)$, is extremely sensitive to $h_{t}$, the top Yukawa coupling. $h_{t}$ is usually the largest dimensionless coupling of the MSSM and is determined at the weak scale by the experimental input for the top quark mass

$$
h_{t}\left(M_{Z}\right)=\frac{\sqrt{2} m_{t}\left(M_{Z}\right)}{v\left(M_{Z}\right) \sin \beta\left(M_{Z}\right)},
$$

where $v\left(M_{Z}\right) \approx 246 \mathrm{GeV}$ is the Higgs vacuum expectation value (VEV) that would be extracted for a single Higgs boson (in terms of the two MSSM Higgs VEVs $v_{1,2}, v=$ $\left.\sqrt{v_{1}^{2}+v_{2}^{2}}\right)$. The running top mass $m_{t}\left(M_{Z}\right)$ is determined from the pole top mass $m_{t}^{\text {pole }}$ by

$$
m_{t}\left(M_{Z}\right)=m_{t}^{\text {pole }}-\Delta m_{t}\left(M_{Z}\right),
$$

where $\Delta m_{t}\left(M_{Z}\right)$ are loop corrections to the top quark mass evaluated at the $\overline{D R}$ renormalisation scale $M_{Z}$. $h_{t}$ is around 1 for typical MSSM spectra (for instance, at parameter point CMSSM10.1.1 [20], $\left.h_{t}\left(M_{Z}\right)=0.90\right)$. Eqs. 1.4,1.5 are used, along with the other RGEs of the MSSM, to evolve $m_{H_{1,2}}^{2}$ along with the other soft SUSY breaking parameters to $M_{S U S Y}$, where the Higgs potential is minimised and Eq. 1.3 is applied. The region of successful EWSB is given by $\mu\left(M_{S U S Y}\right)^{2}>0$ in Eq. 1.3, i.e. (since $\tan \beta>1$ for models

\footnotetext{
${ }^{1}$ This prescription at least ensures that the dominant terms do not involve large logarithms.
} 
in which the lightest $\mathrm{CP}$ even Higgs is heavy enough to satisfy experimental constraints, $\tan 2 \beta<0)$,

$$
\left[m_{H_{2}}^{2}\left(M_{S U S Y}\right) \tan \beta-m_{H_{1}}^{2}\left(M_{S U S Y}\right) \cot \beta\right]<\frac{M_{Z}^{2}}{\tan 2 \beta} .
$$

Eq. 1.8 splits the MSSM parameter space into two regions: one region has successful EWSB whereas the other does not.

In models where we consider the primordial SUSY breaking terms to be set at some higher scale $M_{X}>M_{S U S Y}$, we shall now show that whether or not this condition is satisfied depends upon the other soft SUSY breaking parameters as well as $m_{H_{2}}^{2}\left(M_{X}\right)$, since $m_{H_{i}}^{2}\left(M_{S U S Y}\right)$ is determined by solving Eqs. 1.4,1.5. To leading order in $A=\log \left(M_{X} / M_{S U S Y}\right) /\left(16 \pi^{2}\right)$, we solve them to obtain

$$
\begin{aligned}
m_{H_{2}}^{2}\left(M_{S U S Y}\right)= & m_{H_{2}}^{2}\left(M_{X}\right)-A\left(6 \left[m_{H_{2}}^{2}\left(M_{X}\right)+m_{\tilde{Q}_{3}}^{2}\left(M_{X}\right)+m_{\tilde{u}_{3}}^{2}\left(M_{X}\right)+\right.\right. \\
& \left.\left.A_{t}^{2}\left(M_{X}\right)\right] h_{t}^{2}\left(M_{X}\right)\right) . \\
m_{H_{1}}^{2}\left(M_{S U S Y}\right)= & m_{H_{1}}^{2}\left(M_{X}\right)-A\left(6 \left[\left(m_{H_{1}}^{2}\left(M_{X}\right)+m_{\tilde{Q}_{3}}^{2}\left(M_{X}\right)+m_{\tilde{u}_{3}}^{2}\left(M_{X}\right)+\right.\right.\right. \\
& \left.\left.\left.A_{b}^{2}\left(M_{X}\right)\right] h_{b}^{2}\left(M_{X}\right)\right]\right),
\end{aligned}
$$

where we have neglected terms proportional to electroweak gauge couplings $g_{1,2}^{2}$ in favour of those proportional to $h_{t}^{2}$ or $h_{b}^{2}$. This is a good approximation when $6 A h_{t, b}^{2}\left(M_{X}\right) \ll 1$, which is not the case in the CMSSM. However, it is qualitatively instructive: substituting Eqs. 1.9,1.10 into Eq. 1.8, we obtain an inequality on the soft SUSY breaking parameters evaluated at $M_{X}$ :

$$
\begin{aligned}
m_{H_{2}}^{2}< & \frac{1}{1-6 A h_{t}^{2}}\left\{6 A h_{t}^{2}\left(m_{\tilde{Q}_{3}}^{2}+m_{\tilde{u}_{3}}^{2}+A_{t}^{2}\right)+\frac{M_{Z}^{2}}{\tan \beta \tan 2 \beta}+\right. \\
& \left.\frac{1}{\tan ^{2} \beta}\left[m_{H_{1}}^{2}-6 A h_{b}^{2}\left(m_{\tilde{Q}_{3}}^{2}+m_{\tilde{d}_{3}}^{2}+A_{b}^{2}\right)\right]\right\},
\end{aligned}
$$

where $h_{t, b}, m_{H_{2}}^{2}, m_{\tilde{Q}_{3}}^{2}, m_{\tilde{u}_{3}}^{2}, m_{\tilde{d}_{3}}^{2}, A_{t}$ and $A_{b}$ are all evaluated at $M_{X}$. For large enough values of $m_{H_{2}}^{2}\left(M_{X}\right)$, we see that the inequality is not satisfied and EWSB is not broken successfully. Qualitatively, this behaviour could also be analytically derived in the CMSSM by examining the complicated re-summed logarithm solutions for the soft masses in Ref. [21] in the limit that $h_{b}$ is also neglected in the RGEs (although we do not include them here because they are not especially illuminating).

Much emphasis has been placed in the past upon fine-tuning associated with the region close to the EWSB bound $[12,13]$ where $m_{H_{2}}^{2}\left(M_{S U S Y}\right)$ is somewhat insensitive to the value of $m_{H_{2}}^{2}\left(M_{X}\right)$ assumed. In Ref. [22], a study of the uncertainties of the position of the EWSB boundary in CMSSM parameter space was included, explicitly showing its extreme sensitivity to the top quark mass. Since then, however, the experimental precision with which the top mass has been measured has been much improved, reducing the concomitant uncertainty. The time is ripe for a reanalysis of the uncertainties on EWSB, so that the results may be used for future LHC searches. In section 2, we shall find that the uncertainties are still large despite the improved precision upon the top mass measurement, 
and furthermore we shall demonstrate that they impact on the prospects of discovery. In particular, such uncertainties should be included in the theoretical error when interpreting experimental results in terms of high-scale SUSY breaking models. As a by-product of our study, in section 3, we shall improve the numerical evaluation of the supersymmetric spectrum in this tricky region of parameter space, as determined by SOFTSUSY3.3.4. In section 4, we summarise our results, as well as provide some simple recommendations for interpretations of SUSY searches in terms of high-scale SUSY breaking models that, if followed, will take the uncertainties inherent in EWSB into account.

\section{Uncertainties in Electroweak Symmetry Breaking}

We now quantify the uncertainty inherent in the position of the boundary of successful EWSB, and its effect on interpretations of searches for supersymmetric particles in terms of high-scale SUSY breaking models. This will allow us to suggest some practical steps for interpreters of data that allow such theoretical uncertainties to be taken into account. We expect many models of SUSY breaking in the MSSM to have regions of parameter space where EWSB is not successfully broken. There are however some very constrained MSSM models of SUSY breaking where EWSB is always successful (for example, the large volume string compactified models [23], where the ratio of the universal scalar mass to the universal gaugino mass is constrained to be $1 / \sqrt{3}$ ), or phenomenological models where one fixes $m_{H_{2}}^{2}$ independently of the other soft SUSY breaking parameters, discarding the no EWSB region. However, for most models, we expect problematic regions of unsuccessful EWSB to exist, as in the CMSSM. Bad EWSB regions have been found in minimal anomaly mediation, gaugino mediation and general gauge mediation models to name just a few (see Refs. [24-26], respectively). Here, we shall focus for definiteness on the familiar example of the CMSSM, bearing in mind that very similar effects will be present in most models of SUSY breaking. We expect that our qualitative conclusions will hold in other models which possess such regions in SUSY breaking parameters which are set at a high scale. At the very least, our recommendations of how to quantify such uncertainties should be followed in any model which has a region of parameter space which has no EWSB.

In the CMSSM, the focus point region is close to the region of unsuccessful EWSB and shows extreme sensitivity to the top quark mass through RGE effects. In this region of CMSSM parameter space, $\left(m_{H_{2}}^{2}+m_{\tilde{Q} a_{3}}^{2}+m_{\tilde{u}_{3}}^{2}+A_{t}^{2}\right)$ is large in Eq. 1.4, and $m_{H_{2}}\left(M_{S U S Y}\right)$ in Eq. 1.3 is very sensitive to the value of $h_{t}$. As explained above, $h_{t}\left(M_{Z}\right)$ is in turn fixed by Eq. 1.6, via the running top mass $m_{t}\left(M_{Z}\right)$. This is affected by the pole mass input, which has significant experimental errors (we take $m_{t}=173.5 \pm 1 \mathrm{GeV}[27]$ ) as well as higher order corrections which are not included in the approximation. However, if one fixes SUSY breaking parameters at the electroweak scale, even if there are constraints among the various parameters and a no-EWSB region exists, the dominant uncertainties coming from the top mass (which enter via the RGE) are not present. We note that the uncertainties originating from $m_{t}$ are distinct from those that originate from two-loop (or higher) corrections to the pole masses of supersymmetric particles [28] or higgs bosons [29], which are small (typically a few percent). 
We include all one-loop MSSM contributions to the top mass, as well as the twoloop QCD corrections and two-loop RGE corrections. However, two loop SUSY QCD corrections to the top mass from squarks and gluinos are not taken into account via Eq. 1.7 in SOFTSUSY3.3.4, nor are 3-loop RGE corrections to $h_{t}$. The full MSSM two-loop $\mathcal{O}\left(\alpha_{s}^{2}\right)$ threshold corrections to $m_{t}$ and three-loop RGE effects were calculated numerically in the CMSSM $[30,31]$ to be at the $2-3 \%$ level for $\tan \beta=50$ and $A_{0}=0$. There, it was noticed that in the focus point region, the position of the no EWSB forbidden region moves by around $300 \mathrm{GeV}$ in $m_{0}$. In order to estimate the size of the $\mathcal{O}\left(\alpha_{s}^{2}\right)$ threshold corrections for our sets of parameters, we use Eq. (62) of Ref. [32], which gives the two-loop SUSY QCD contribution in the limit that the squark and gluino masses are all of equal mass $M$ :

$$
\begin{aligned}
\left(\frac{\Delta m_{t}(Q)}{m_{t}(Q)}\right)_{2 L S Q C D}= & \frac{4}{3}\left(\frac{\alpha_{s}}{4 \pi}\right)^{2}\left\{\frac{47}{3}+20 \ln \left(\frac{M^{2}}{Q^{2}}\right)+6 \ln \left(\frac{M^{2}}{Q^{2}}\right) \ln \left(\frac{M^{2}}{m_{t}^{2}}\right)\right. \\
& +\frac{4}{3}\left[\frac{23}{24}-\frac{13}{6} \ln \left(\frac{M^{2}}{Q^{2}}\right)+\frac{1}{2} \ln ^{2}\left(\frac{M^{2}}{Q^{2}}\right)-3 \ln \left(\frac{M^{2}}{Q^{2}}\right) \ln \left(\frac{m_{t}^{2}}{Q^{2}}\right)\right] \\
& +3\left[\frac{175}{72}+\frac{41}{6} \ln \left(\frac{M^{2}}{Q^{2}}\right)-\frac{1}{2} \ln ^{2}\left(\frac{M^{2}}{Q^{2}}\right)-2 \ln \left(\frac{M^{2}}{Q^{2}}\right) \ln \left(\frac{m_{t}^{2}}{Q^{2}}\right)\right] \\
& +\frac{X_{t}}{M}\left[-4-8 \ln \left(\frac{M^{2}}{Q^{2}}\right)\right]+\frac{4}{3} \frac{X_{t}}{M}\left[\frac{7}{3}-\frac{11}{3} \ln \left(\frac{M^{2}}{Q^{2}}\right)+3 \ln \left(\frac{m_{q}^{2}}{Q^{2}}\right)\right] \\
& \left.+3 \frac{X_{t}}{M}\left[-\frac{8}{3}+4 \ln \left(\frac{M^{2}}{Q^{2}}\right)\right]\right\},
\end{aligned}
$$

where $X_{t}=A_{t}-\mu \cot \beta$. The squark masses are typically much larger than the gluino masses in the focus point region, and so this may be a bad approximation to the actual correction. However, we may estimate the size of the theoretical error roughly by estimating the size of Eq. 2.1 across parameter space (i.e. assuming that the squark and gluino masses are all equal to the average of the dimensionally reduced values of both stop masses and the gluino mass).

In our numerical analysis, we interpolate a grid of $m_{0}$ and $M_{1 / 2}$ values for $A_{0}=0$ and $m_{b}\left(m_{b}\right)=4.18 \mathrm{GeV}$ by using SOFTSUSY3.3.4 [8]. Fig. 1 shows the estimated size of the higher order correction, as determined in the way described above. Across the parameter space, we see that $\left|\left(\Delta m_{t}\right)_{2 L S Q C D}\left(M_{S U S Y}\right)\right|<0.8 \mathrm{GeV}$. We may approximately model the effect of this theoretical uncertainty by absorbing it into the pole mass input uncertainty. If one wanted to do this more precisely, one would renormalise $\left(\Delta m_{t}\right)_{2 L S Q C D}$ from $M_{S U S Y}$ back to $M_{Z}$, where the running top mass is matched to the pole mass. However, the difference between including $\left(\Delta m_{t}\right)_{2 L S Q C D}\left(M_{S U S Y}\right)$ and $\left(\Delta m_{t}\right)_{2 L S Q C D}\left(M_{Z}\right)$ is a threeloop effect, which we neglect. It is possible that we have underestimated the overall size of the theoretical uncertainty: after all, we have not included possible effects from 3-loop RGEs [33] or from 2-loop threshold corrections to $m_{t}$ [32]. We shall conservatively add the $0.8 \mathrm{GeV}$ theoretical uncertainty linearly to the $2 \sigma$ measurement errors upon the input value of $m_{t}$ in order to estimate the total uncertainty in EWSB.

Having found and quantified the error due to the dominant theoretical uncertainty, we may combine it with the $m_{t}$ measurement errors in order to see the effect on the location 


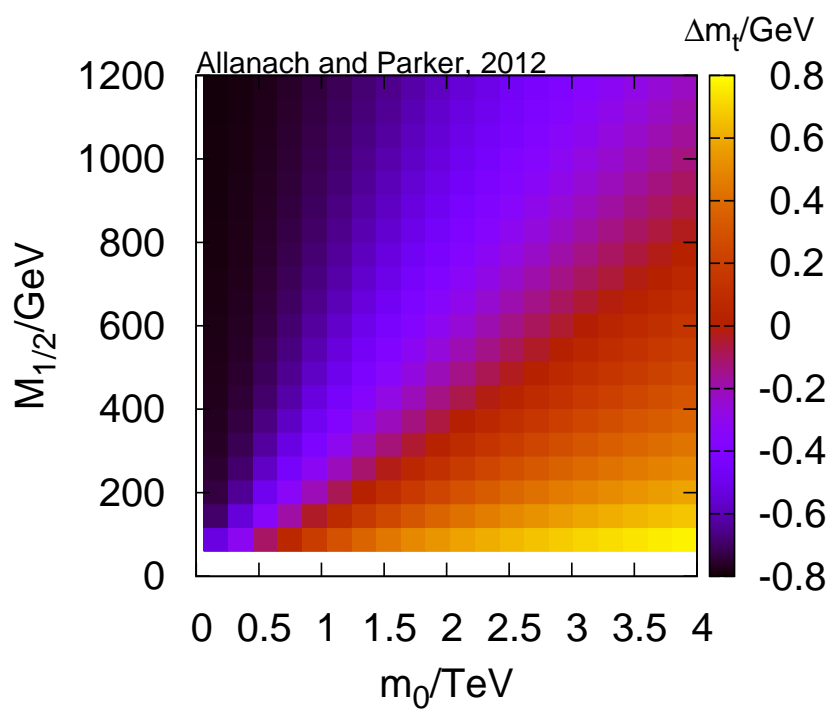

Figure 1. Estimated size of higher order correction (2 loop SUSY QCD correction) $\left(\Delta m_{t}\right)_{2 L S Q C D}\left(M_{S U S Y}\right)$ in the CMSSM for $\tan \beta=10$ and $A_{0}=0$. The correction has a size as measured by reference to the colour bar on the right hand side.

of the EWSB boundary. The result is shown in Fig. 2 for $A_{0}=0$, (a) $\tan \beta=10$ and (b) $\tan \beta=50$. By varying the experimental uncertainty on $m_{t}$ within $2 \sigma$ of its central value, we obtain the yellow (lighter) shaded region showing the uncertainty in the boundary's location. The curves marked 'EWSB boundary' are, from left to right, for $m_{t}=171.5$ $\mathrm{GeV}, m_{t}=173.5 \mathrm{GeV}$ and $m_{t}=175.5 \mathrm{GeV}$, respectively. Varying $m_{t}$ by a further 0.8 $\mathrm{GeV}$ in either direction, we obtain the grey bands, providing an estimate of the theoretical uncertainty. We also show the expected $5 \sigma$ discovery reaches for 4 jets plus (0-3) leptons for a $14 \mathrm{TeV}$ LHC and $1 \mathrm{fb}^{-1}$ of luminosity from Ref. [34]. We see that the location of the boundary is uncertain to a huge $\sim 2 \mathrm{TeV}$ in $m_{0}$, and that the discovery regions extend well into the region of EWSB uncertainty, irrespective of the size of $\tan \beta$, even for only 1 $\mathrm{fb}^{-1}$ of collected data. We also note that the $2 \sigma$ exclusion contours will be at much larger values of $m_{0}$ and $M_{1 / 2}$, and so the problem of the uncertain EWSB boundary will be more acute for exclusion than for discovery. This combined with larger $14 \mathrm{TeV}$ data sets implies that the EWSB uncertainty is going to be very relevant for SUSY searches in the future.

In Fig, 3, we show that the size of the uncertainty can make a large difference to the production cross-sections of squark (anti-)squark $\sigma_{s s}$ and (anti-)squark gluino $\sigma_{s g}$ as calculated by PROSPINO2 [35], using the SUSY Les Houches Accord [36] to transfer information about the MSSM spectrum. The solid lines show the limits of the uncertainty, as defined by both the experimental $2 \sigma m_{t}$ uncertainties and the $0.8 \mathrm{GeV}$ theoretical error acting in tandem. If, for instance, we assume the CMSSM and constrain $M_{1 / 2}=400$ $\mathrm{GeV}$ from other measurements, squarks may be highly visible at the LHC (when jointly produced with gluinos as shown in Fig. 2b) with large production cross-sections (100 fb), or they may require a luminosity upgrade ( $1 \mathrm{fb}$ or less) for discovery, depending upon the locus of the boundary. Of course, this would not affect SUSY discovery because discovery would be dominated by production of the lighter sparticles. However, the interpretation of 
$\tan \beta=10$

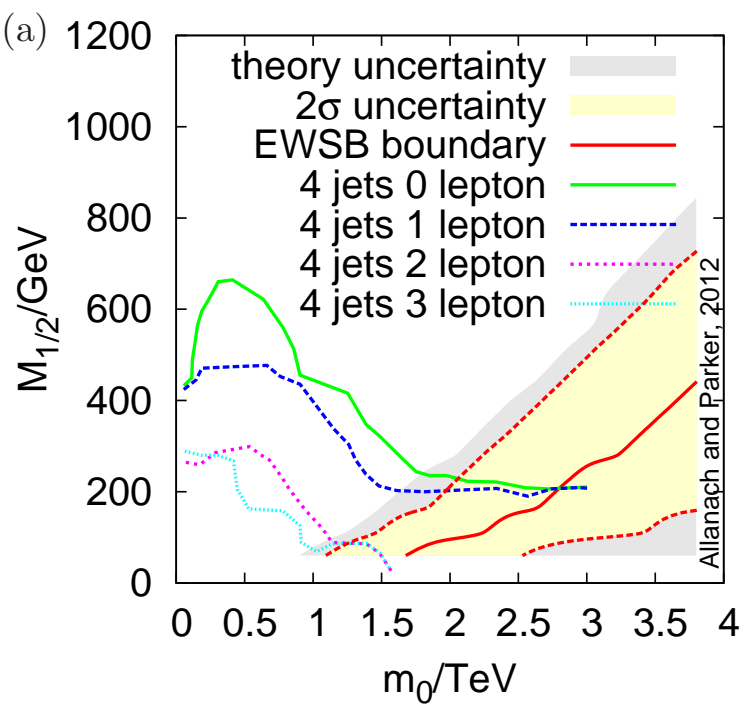

$\tan \beta=50$

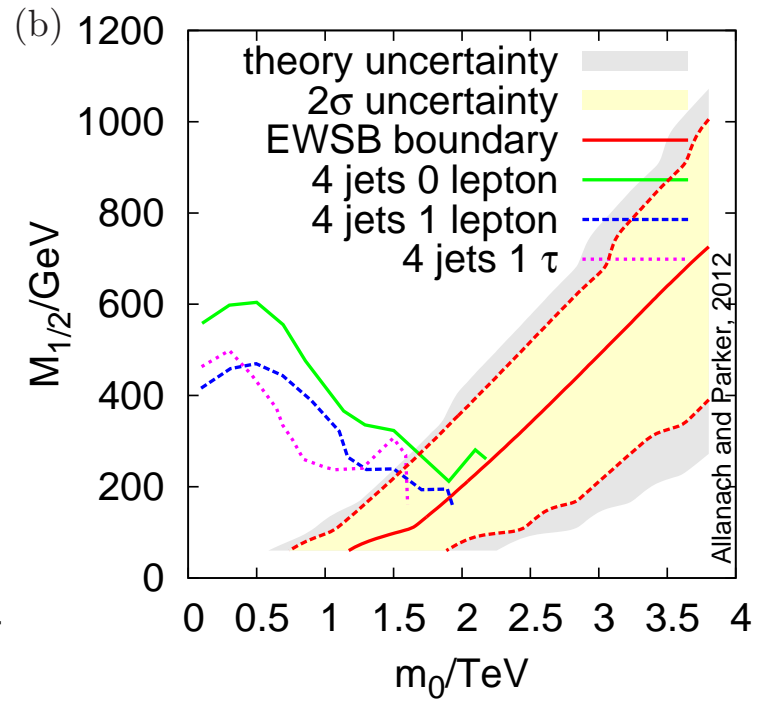

Figure 2. Uncertainties in the position of the EWSB boundary compared to the expected $5 \sigma$ ATLAS $14 \mathrm{TeV}$ CMSSM search reach for $1 \mathrm{fb}^{-1}$ from Ref. [34]. The region below the 'EWSB boundary' is theoretically inaccessible: the uncertainty in the position of this region due to $2 \sigma$ variations in $m_{t}$ is shown by the yellow band. The theoretical uncertainty is marked by the grey region and estimated as detailed in the main body of the paper.

supersymmetric signals in terms of a high-scale SUSY breaking model would be obscure unless the uncertainties are taken into account. For example, it may be that the values of $m_{0}$ and $M_{1 / 2}$ inferred from measurements of $\sigma_{s g}$ and other processes appear to be in the EWSB region. One would then erroneously rule the CMSSM out. When searches are interpreted near the boundary of EWSB therefore, in a high-scale SUSY breaking model such as the CMSSM, it is important to generate default SUSY spectra using a $m_{t}$ input value that is $2 \sigma$ higher than the central value, so that the high end of the uncertain region is included in the search. Increasing $m_{t}$ has a negligible effect on the gluino and first or second generation squark masses, so the production cross-sections should not change much. However, we show below that it has an effect on the predicted lightest neutralino mass in the high-scale model. $m_{t}$ does have a larger effect on stop searches and so here, one would need to check the effect's magnitude by taking the variation of the stop masses from $m_{t}$ into account.

On the other hand, we know that $\mu \rightarrow 0$ at the EWSB boundary, affecting the mass of the lightest supersymmetric particle (LSP), here assumed to be the lightest neutralino. The MSSM Lagrangian contains the neutralino mass matrix as $-\frac{1}{2} \tilde{\psi}^{0 T} \mathcal{M}_{\tilde{\psi}^{0}} \tilde{\psi}^{0}+$ h.c., where 


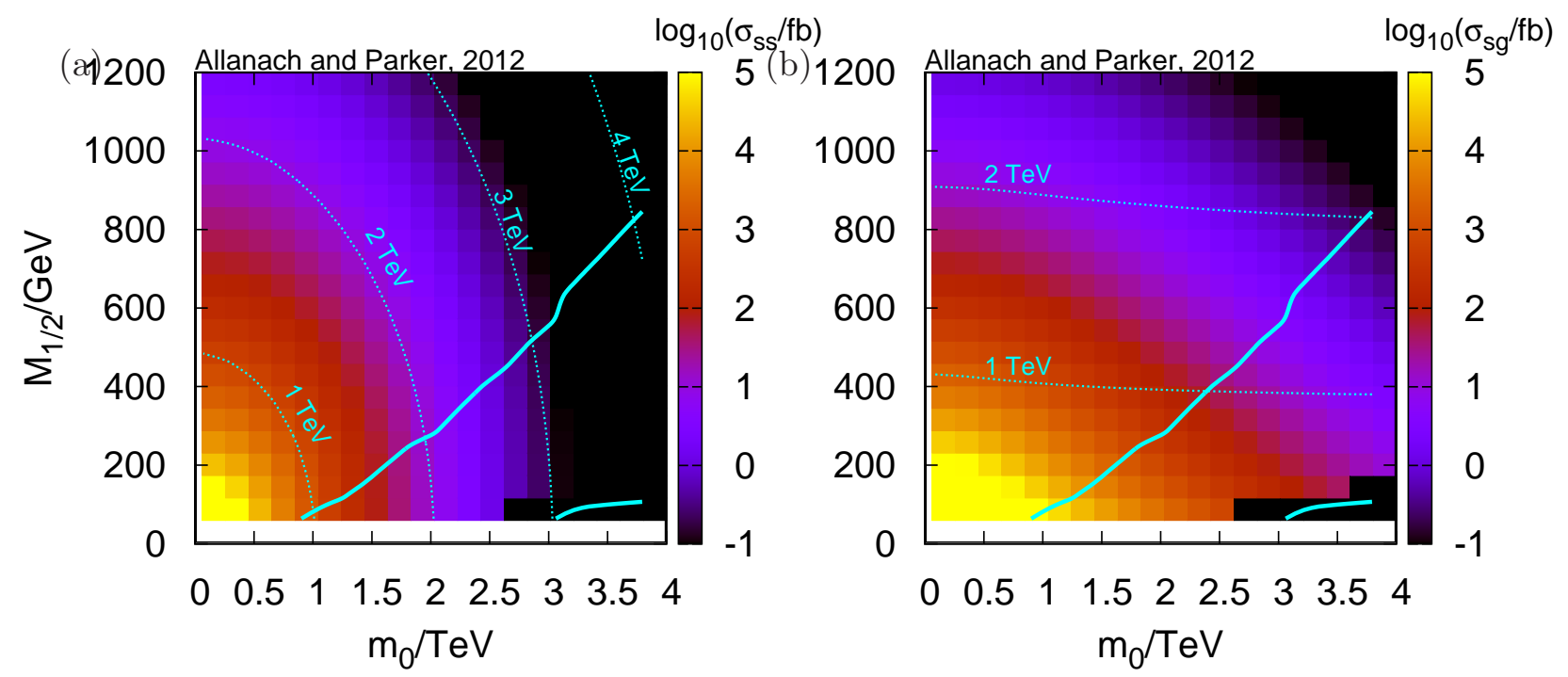

Figure 3. Uncertainties in EWSB correlated with (a) squark-(anti-)squark and (b) squark gluino production cross-section at the $14 \mathrm{TeV}$ LHC for $\tan \beta=10$ and $A_{0}=0$. The two solid lines show the total uncertainty on the position of the boundary of EWSB. The total production cross-sections (with no cuts applied) are shown by reference to the colour bar on the right hand side. The dotted lines show (a) iso-contours of $m_{\tilde{q}}$ and (b) iso-contours of $m_{\tilde{g}}$, as labelled.

$\tilde{\psi}^{0}=\left(-i \tilde{b},-i \tilde{w}^{3}, \tilde{h}_{1}, \tilde{h}_{2}\right)^{T}$ and, at tree level,

$$
\mathcal{M}_{\tilde{\psi}^{0}}=\left(\begin{array}{cccc}
M_{1} & 0 & -M_{Z} c_{\beta} s_{W} & M_{Z} s_{\beta} s_{W} \\
0 & M_{2} & M_{Z} c_{\beta} c_{W} & -M_{Z} s_{\beta} c_{W} \\
-M_{Z} c_{\beta} s_{W} & M_{Z} c_{\beta} c_{W} & 0 & -\mu \\
M_{Z} s_{\beta} s_{W} & -M_{Z} s_{\beta} c_{W} & -\mu & 0
\end{array}\right) .
$$

We use $s$ and $c$ for sine and cosine, so that $s_{\beta} \equiv \sin \beta, c_{\beta} \equiv \cos \beta$ and $s_{W}\left(c_{W}\right)$ is the sine (cosine) of the weak mixing angle. The condition $\mu \rightarrow 0$ (at the EWSB boundary) results in a lightest neutralino mass (equal to the smallest eigenvalue of Eq. 2.2) that is zero at tree-level $^{2}$. Including loop corrections to the neutralino mass matrix, the neutralino LSP is still very light, typically less than $1 \mathrm{GeV}$. As the position of the EWSB boundary is so uncertain, the position of this negligible neutralino mass occurs for very different values of $m_{0}$. Since $m_{0}$ controls the squark mass, the correlation between squark and LSP mass picks up large uncertainties. We illustrate the problem by scanning along the $M_{1 / 2}=300 \mathrm{GeV}$ line for various different values of $m_{t}$, including an estimate of the theoretical uncertainty originating from higher order corrections to $m_{t}$ as described above. From Fig. 4a, we see the large uncertainty induced in the lightest neutralino mass along the line. We see

\footnotetext{
${ }^{2}$ One can easily see this by the observation that adding $\tan \beta$ multiplied by the third column to the fourth column leads to a column of zeroes in the $\mu \rightarrow 0$ limit. The resulting matrix, which has a determinant of $\tan \beta$ multiplied by the determinant of the original matrix, must therefore have a zero determinant and therefore possesses a zero eigenvalue.
} 

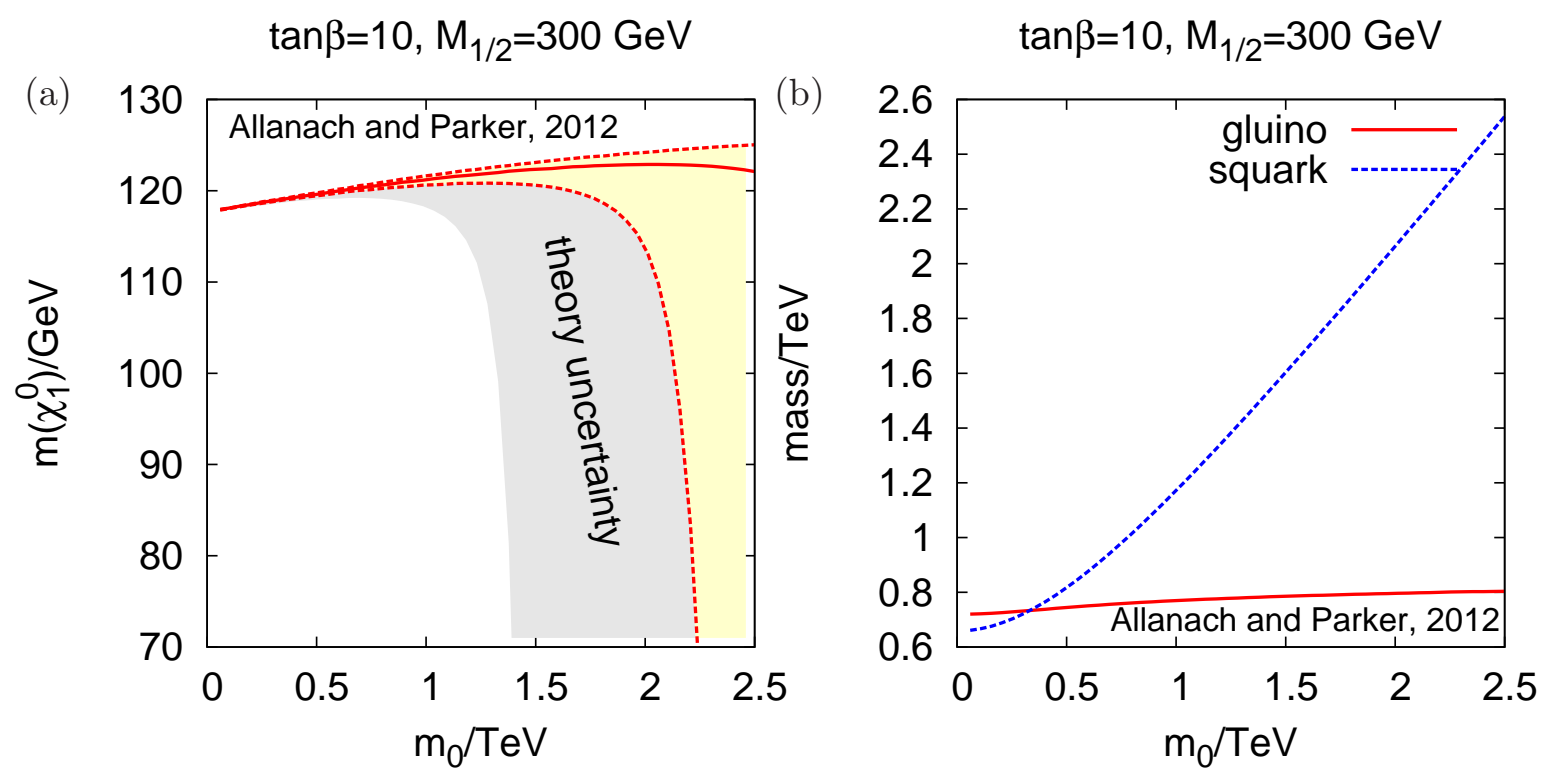

Figure 4. Evolution in certain SUSY particle masses approaching the EWSB boundary for $\tan \beta=$ $10, M_{1 / 2}=300 \mathrm{GeV}$ and $A_{0}=0$ in the CMSSM. (a) shows uncertainties in the CMSSM predicted lightest neutralino mass. The solid line has $m_{t}=173.5 \mathrm{GeV}$, whereas the upper and lower dashed lines have $m_{t}=175.5 \mathrm{GeV}$ and $m_{t}=171.5 \mathrm{GeV}$, respectively. Additional uncertainty coming from the higher order theoretical uncertainty in $m_{t}$ via RGE effects is marked by the grey region. (b) shows values of the squark and gluino masses as a function of $m_{0}$ along the $M_{1 / 2}=300 \mathrm{GeV}$ line.

from Fig. $4 \mathrm{~b}$ that the high $m_{0}$ region where the neutralino mass is particularly uncertain corresponds to large values of the squark masses $>1.5 \mathrm{TeV}$. Here, the classic jets plus missing transverse momentum signature will come primarily from $\tilde{g} \tilde{g}$ production, where $\tilde{g}$ decays through an off-shell squark into two jets and the neutralino LSP. In the centre of mass frame of the 700-800 GeV gluino, the gluino must share its rest mass energy between the two jets and the lightest neutralino. Having higher neutralino masses therefore has the effect of reducing the $p_{T}$ of the jets, on average. Since experimental analyses impose lower limits on the jet $p_{T} \mathrm{~s}$ in the cuts, the efficiency of the cuts will decrease for higher neutralino masses. We therefore expect the theoretical uncertainty in the neutralino mass to induce a non-negligible uncertainty in the cut efficiency.

In Figure 5 we plot data on the acceptance times efficiency $(A \times \varepsilon)$ for the ATLAS search based on all hadronic final states with missing energy [1]. These data, for the 7 $\mathrm{TeV}$ sample, are available in the auxiliary information of Ref. [1]. The data correspond to a model in which the gluino decays directly to jets and LSP, with the gluino mass fixed at $1050 \mathrm{GeV}$, close to the EWSB boundary. The other SUSY particle masses are set high enough for them to decouple. The figures show the fraction of signal events which would pass the experimental selections (based largely on the $p_{T}$ of the jets, and the missing transverse momentum, and also be successfully reconstructed after detection, as a function of the LSP mass. Figures $5 \mathrm{a}$ and $\mathrm{b}$ show the lowest and highest effective mass selections for each jet multiplicity, respectively. The value of $A \times \varepsilon$ drops rapidly at high LSP mass 
(a)

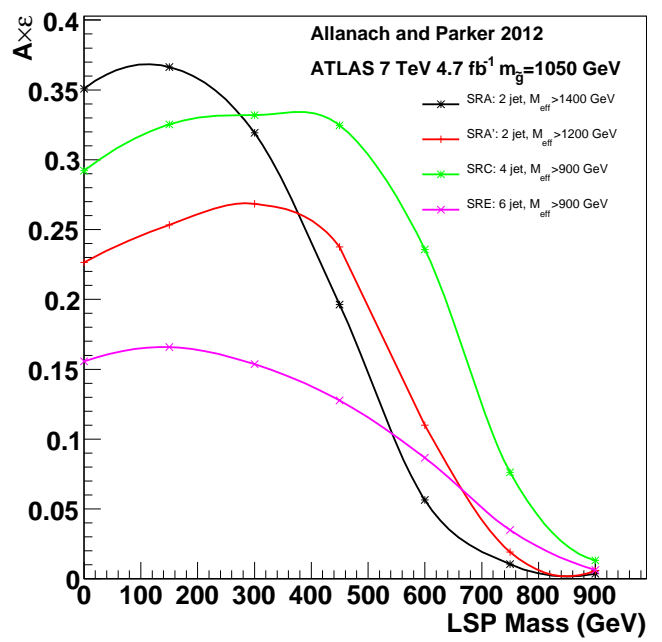

(b)

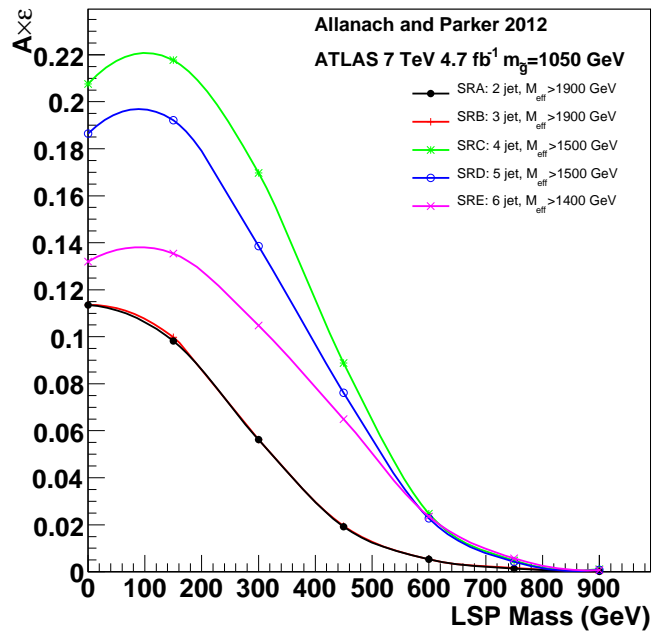

Figure 5. Acceptance times efficiency $A \times \epsilon$ for signal events selected by the ATLAS supersymmetry search [1], using the signature of missing energy with hadronic jets. The different signal regions correspond to final state jet multiplicities (n) between 2 and 6, as shown in the key. The signal regions are selected with minimum values of $m_{\mathrm{eff}}$, the scalar sum of the $p_{T} \mathrm{~s}$ of the $\mathrm{n}$ leading jets (appropriate to each signal region) with the missing energy. The lowest available $m_{\text {eff }}$ selection is shown in (a), and the highest in (b) for various jet multiplicities.

for all of the signal regions. This is expected, since the fraction of the initial squark or gluino energy passing into the observable jets is reduced as the LSP mass rises, and hence the fraction passing the $p_{T}$ selections drops. For the lowest multiplicity final states (SRA), with the higher effective mass selection, the effect can be seen even at the lowest LSP masses, with $A \times \varepsilon$ reducing from $11.7 \%$ to $9.8 \%$ as the LSP mass rises from 0 to $150 \mathrm{GeV}$. This represents a change in signal event rate of $17 \%$, a non-negligible uncertainty. In the lower effective mass samples, $A \times \varepsilon$ rises in the same LSP mass range in some cases (an effect already noted in Ref. [37]). For example, for SRA', it increases from $23.5 \%$ to $26.1 \%$, increasing the signal rate by $11 \%$, and continues to rise for LSP masses up to $300 \mathrm{GeV}$. This may be due to the behaviour of the cut on the ratio of the missing energy to $m_{\mathrm{eff}}$ applied in this analysis, since the two distributions will evolve in slightly different ways as the LSP mass changes. These effects can be expected to continue to occur at higher centre of mass energies, with the final drop in $A \times \epsilon$ moving to higher LSP masses.

Even if the high-scale SUSY breaking interpretation of an experiment's sparticle search is largely independent of the uncertainty induced in the LSP mass, it is possible that other sparticle masses in the signal sparticle cascade decay chains are highly dependent and cause large theoretical uncertainties in signal cut efficiencies. In particular, chargino masses are highly dependent on $\mu\left(M_{S U S Y}\right)$. Also, third generation sparticles may be highly dependent on $\mu\left(M_{S U S Y}\right)$ in some (but not all) regions of parameter space. Extra care to quantify the theoretical error should be taken in searches for cascade chains involving these sparticles, particularly when they are on-shell (since the mass splittings of on-shell decays may have a particularly large effect on the kinematics of the decay products). In order to take 


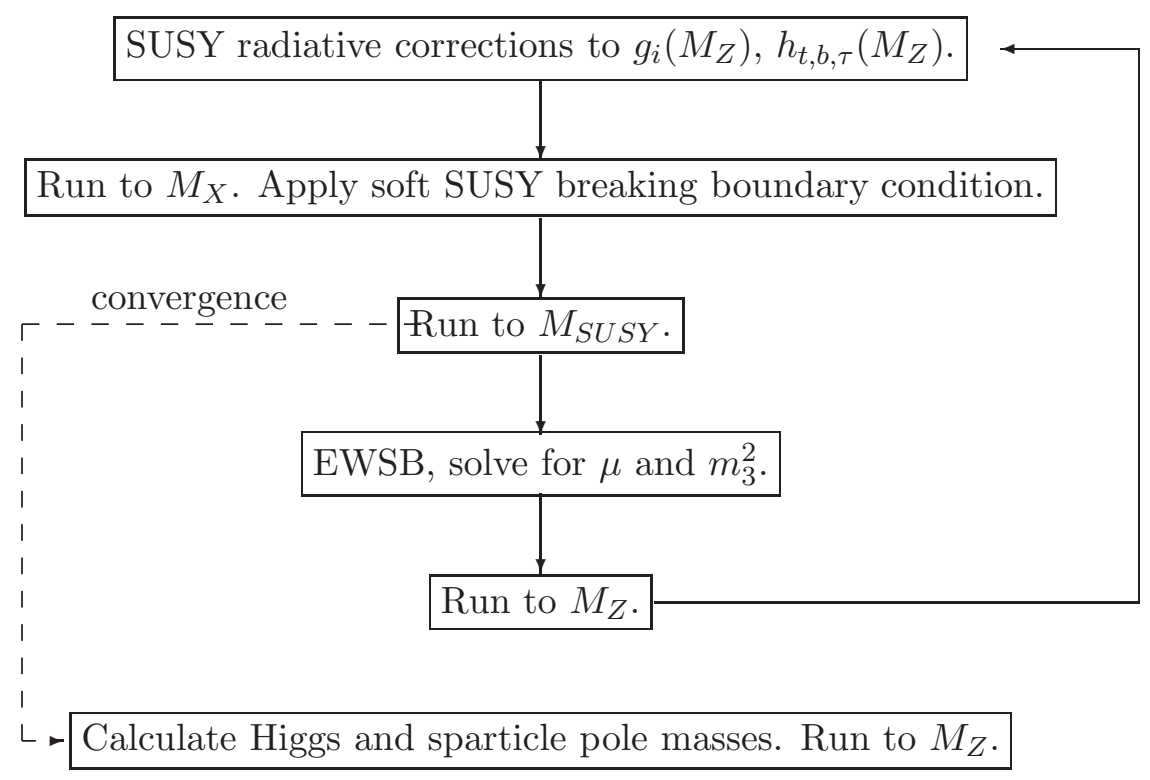

Figure 6. Iterative algorithm used to calculate the SUSY spectrum. The initial step is the uppermost one. $M_{S U S Y}$ is the scale at which the EWSB conditions are imposed, as discussed in the text. $M_{X}$ is the scale at which the high energy SUSY breaking boundary conditions are imposed.

such uncertainties into account, varying $m_{t}$ within its $2 \sigma$ limits, including the size of the shift from higher order corrections (estimated here to be $0.8 \mathrm{GeV}$ ) in the estimate of the uncertainty will suffice, since the various relevant sparticle masses will vary according to the concomitant theoretical error.

\section{Convergence}

As one approaches the boundary of EWSB in the parameter space of high-scale supersymmetry breaking models, one finds that the various spectrum generators start to struggle to produce numerically stable results. This is because of the extreme sensitivity of the RGEs and boundary conditions, but is not a problem if one is only fixing the SUSY breaking parameters at the weak scale. SOFTSUSY3.3.4 is essentially solving coupled, non-linear ordinary differential equations with two sets of boundary conditions: one set on the gauge couplings $g_{i}$, the Yukawa couplings $h_{t, b, \tau}$ and the Higgs potential parameters $\mu$ and $m_{3}^{2}$ at the weak scale, and the other set on the soft SUSY breaking parameters at the high scale $M_{X}$. SOFTSUSY3.3.4 uses an iterative algorithm, as shown in Fig. 3. For SOFTSUSY3.3.4 to report that it has solved the problem, a convergence criterion must be satisfied: the values of the $\overline{D R}$ sparticle masses of the current iteration must all be identical to the previous iteration's, to some fractional accuracy $\epsilon$. Here, $\epsilon=10^{-3}$. Near the boundary of EWSB, $\mu\left(M_{S U S Y}\right)$ and $m_{3}\left(M_{S U S Y}\right)$ become extremely dependent upon the precise value of $h_{t}\left(M_{S U S Y}\right)$. However, $h_{t}\left(M_{S U S Y}\right)$, which is dependent on $m_{t}\left(M_{S U S Y}\right)$, varies from iteration to iteration because the loop corrections depend upon the sparticle masses, which 


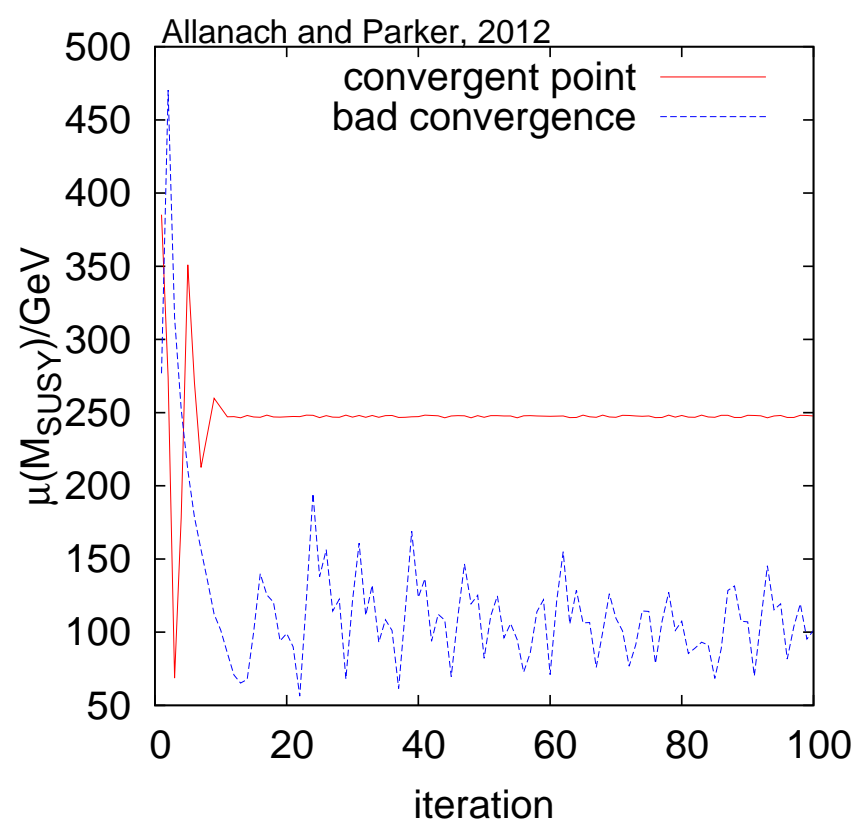

Figure 7. Convergence of the numerical solution to EWSB Higgs potential minimisation conditions near the boundary of electroweak symmetry breaking in the CMSSM using SOFTSUSY3.3.4. We have set $\epsilon=10^{-3}, m_{t}=173.5 \mathrm{GeV}, \tan \beta=10, A_{0}=0, M_{1 / 2}=337.5 \mathrm{GeV}$ and $m_{0}=3000 \mathrm{GeV}$ for the 'convergent point', or $m_{0}=3400 \mathrm{GeV}$ for the 'bad convergence' point.

also vary from iteration to iteration. In parameter space that is sufficiently near to the boundary of EWSB, this numerical iteration noise becomes too great for the program to find a solution, as illustrated in Fig. 7. We show two points in CMSSM parameter space, one with bad convergence, and one with good convergence. We display the predicted value of $\mu\left(M_{S U S Y}\right)$, which is one of EWSB variables that is most sensitive. We see that after 10 iterations, the convergent point has found a stable solution for $\mu\left(M_{S U S Y}\right)$, whereas there is no indication that the point with bad convergence would ever converge. In practice, if no convergence is achieved after 30 iterations, SOFTSUSY3.3.4 halts the calculation reporting a 'no convergence' error.

If one's scans in parameter space are sufficiently coarse, one may not be aware of this problem, as the region of no convergence is fairly thin in the $m_{0}-M_{1 / 2}$ plane. We zoom in on the plane in Fig. 8 in order to investigate it. Fig. 8a shows that the yellow (light) regions of bad convergence are prevalent close to the boundary of no EWSB. Using Eq. 1.3 to predict $M_{Z}$ for the numerical solution that issues from SOFTSUSY3.3.4 (whether or not convergence is reached), we plot its value in the zoomed region in Fig. 8b. We see that throughout this zoomed region, despite the fact that central experimental value of $M_{Z}$ has been used at each iteration to constrain the model, the large sensitivity spoils the prediction after each iteration and the predicted value is far from the central one of $M_{Z}^{\text {cent }}=91.1876$ GeV [27]. The diagonal line in Fig. 8a across parameter space shows the line taken in Fig. 9 to illustrate how how the numerical spectral predictions of SOFTSUSY3.3.4 vary 


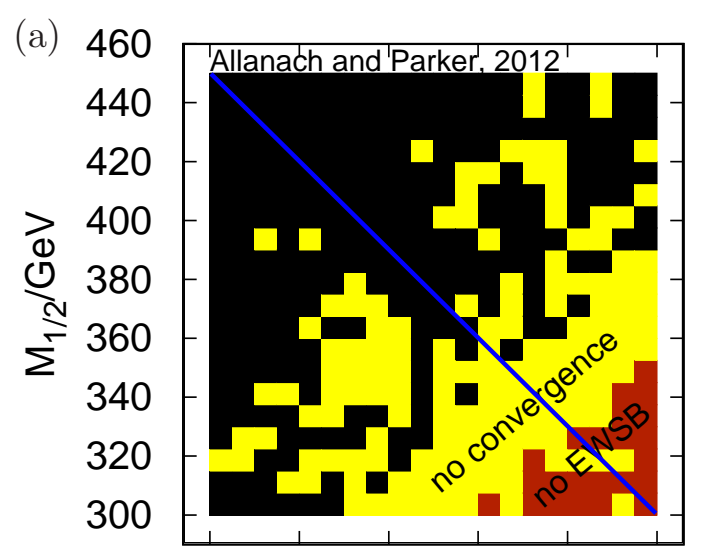

$\begin{array}{llllll}3 & 3.1 & 3.2 & 3.3 & 3.4 & 3.5\end{array}$ $\mathrm{m}_{0} / \mathrm{TeV}$

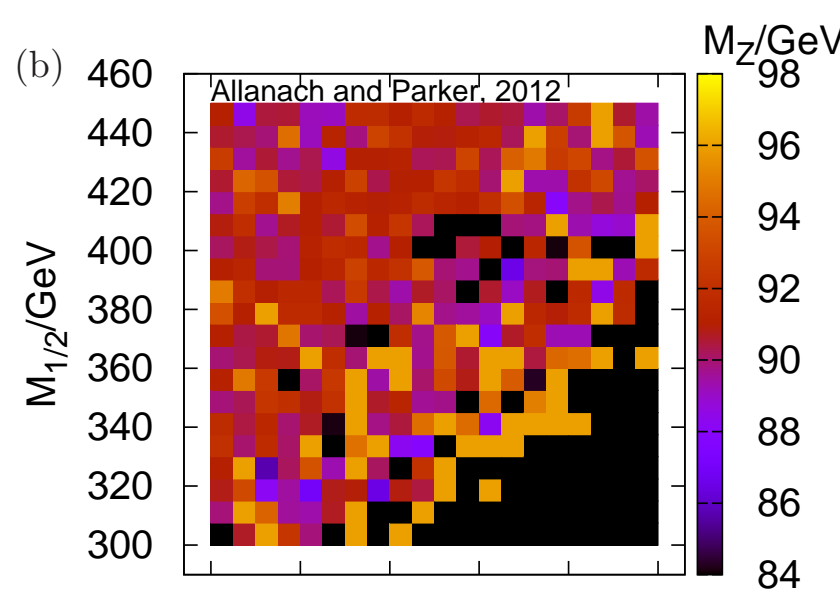

$\begin{array}{llllll}3 & 3.1 & 3.2 & 3.3 & 3.4 & 3.5\end{array}$

$\mathrm{m}_{0} / \mathrm{TeV}$

Figure 8. Zoom of CMSSM focus point parameter space as predicted by SOFTSUSY3.3.4 for $A_{0}=0, m_{t}=173.5 \mathrm{GeV}$ and $\tan \beta=10$ : (a) shows the region of successful EWSB (black), bad numerical convergence (yellow) and no EWSB (red). The diagonal line shows the parameter line taken to illustrate non-convergence in Fig. 9. (b) displays the predicted value of $M_{Z} / G e V$, as measured by the colour bar to the right hand side.

while travelling through this problematic region of parameter space. Each line represents a different sparticle mass divided by some constant number of $\mathrm{GeV}$, chosen so that the ratios are all similar and appear near to each other on the same plot. We see that the gluino, stop and pseudo-scalar Higgs masses have a smooth behaviour as the region of no EWSB is approached. However, we see that the neutralino LSP mass, which depends upon $\mu\left(M_{S U S Y}\right)$, becomes erratic close to the boundary, corresponding to numerical problems and non-convergence of the iteration algorithm. Using the numerical solution at the inferred boundary but substituting $\mu=0$ into the one loop level neutralino mass matrix, we infer a mass of $0.1 \mathrm{GeV}$ for the neutralino. The neutralino line would be at $m / M \approx 0.01$ at this point, rather than around 0.6. Some of the erratic non-convergent behaviour can be hidden behind the LEP chargino bound of $M_{\chi^{ \pm}}>104 \mathrm{GeV}$ [38], but still some noise remains unhidden.

We improve the algorithm in several ways: firstly instead of just 30 iterations, we allow a maximum of 100. This helps the program achieve convergence at some parameter points. Secondly, we specify that the predicted value of $M_{Z}^{\text {pole }}$ must be close enough to the empirical central value for convergence to be possible. Thus, the convergence criteria are enlarged to include the constraint that $\left|M_{Z}^{\text {pole }} / M_{Z}^{\text {cent }}-1\right|<\epsilon$ within the first 10 iterations, or $\left|M_{Z}^{\text {pole }} / M_{Z}^{\text {cent }}-1\right|<10 \epsilon$ after the first 10 iterations. Thirdly, there are some sub iterations (for example for the solution of Eq. 1.3 for $\mu\left(M_{S U S Y}\right)$ once loop corrections are included) which are required to converge to much higher accuracy, given the sensitivity of each iteration to the underlying parameters. Finally, we further smooth the iteration of $\mu\left(M_{S U S Y}\right)$ if the parameter point is proving difficult. Between iteration 11 and 20 , the average of the value of $\mu_{\text {old }}$ from the previous iteration and the one predicted by the EWSB 


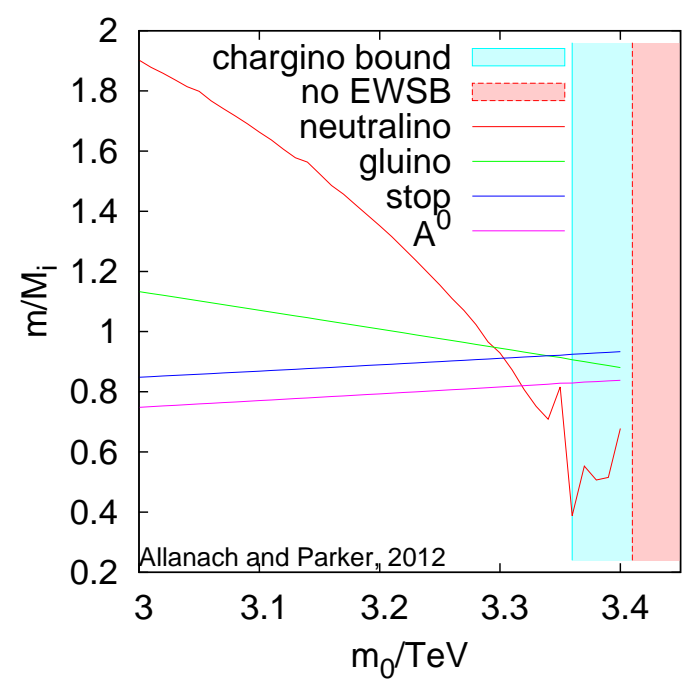

Figure 9. SOFTSUSY3.3.4 convergence approaching the boundary of EWSB in the CMSSM for $m_{t}=173.5 \mathrm{GeV}$. The plot shows the blue line in Fig. 8 (i.e. $M_{1 / 2}$ is varying across the plot as well as $m_{0}$ ). We have scaled each $i^{t h}$ particle mass by a constant mass $M_{i}$ for clarity. The region where electroweak symmetry is not satisfactorily broken is denoted 'no EWSB'. $m_{\chi_{1}^{ \pm}}>104 \mathrm{GeV}$, in contravention of the LEP bound, in the area marked 'chargino bound'.

conditions acting on the current iteration $\mu_{\text {ews }}$ is taken. Between iteration 21 and 30, SOFTSUSY3.3.6 takes $\mu\left(M_{S U S Y}\right)=0.2 \mu_{\text {ews }}+0.8 \mu_{\text {old }}$, whereas after iteration 31 , it takes $\mu\left(M_{S U S Y}\right)=0.1 \mu_{\text {ews }}+0.9 \mu_{\text {old }}$. We find that this helps dampen the oscillations apparent in the points that exhibit poor convergence properties such as the 'bad convergence' line in Fig. 7. The resulting improvement in convergence is shown in Fig. 10. The region of no convergence has significantly diminished, and become much more regular as compared to Fig. 8a. Also, in the region of successful EWSB, $M_{Z}$ is predicted to be much closer to $M_{Z}^{\text {cent }}$, signalling a good solution to the EWSB conditions. These improvements are implemented in an improved version of SOFTSUSY (version 3.3.6) [8].

\section{Summary and Conclusions}

We have studied the uncertainties associated with the boundary of viable parameter space in constrained high-scale SUSY mediation models of the MSSM coming from successful EWSB. Even with the current accurate measurements of $m_{t}$, there is an enormous $2 \mathrm{TeV}$ variation in the CMSSM boundary's position as measured by the soft SUSY breaking scalar mass input $m_{0}$. There is also a theoretical uncertainty associated with higher order corrections to the top mass, equivalent to around $0.8 \mathrm{GeV}$. Uncertainties on $m_{t}$ translate into an uncertainty on $h_{t}$, which the RGEs are very sensitive to, and to which, ultimately, the EWSB region becomes uncertain. There is therefore no concomitant EWSB uncertainty in simplified models or other low-scale models such as the phenomenological MSSM. As well as providing an uncertainty on the boundary of viable parameter space, there is also, 


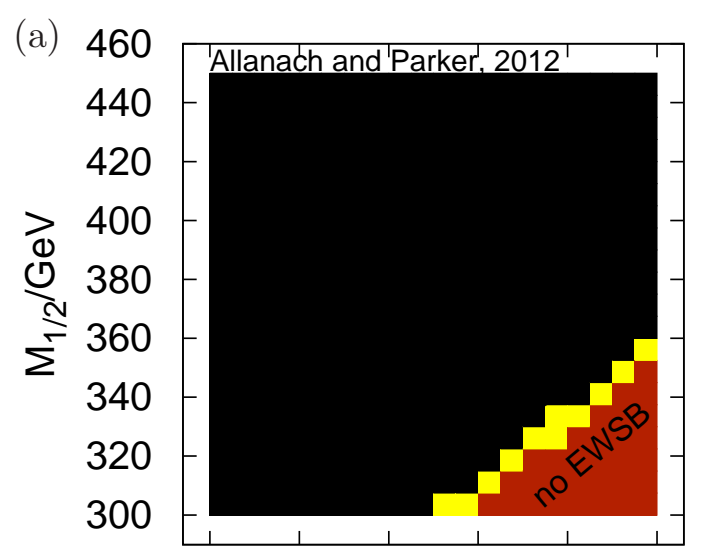

$\begin{array}{llllll}3 & 3.1 & 3.2 & 3.3 & 3.4 & 3.5\end{array}$ $\mathrm{m}_{0} / \mathrm{TeV}$

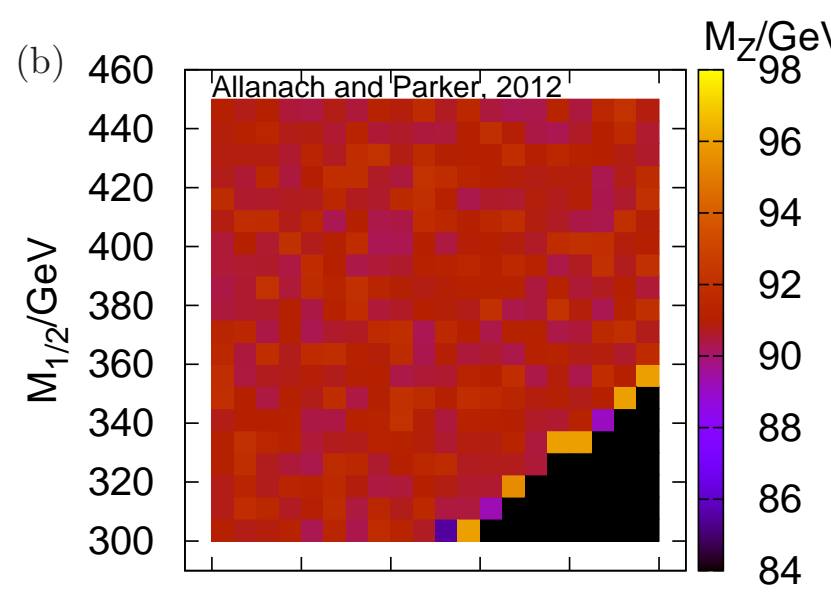

$\begin{array}{llllll}3 & 3.1 & 3.2 & 3.3 & 3.4 & 3.5\end{array}$

$\mathrm{m}_{0} / \mathrm{TeV}$

Figure 10. Zoom of CMSSM focus point parameter space with the improved algorithm in SOFTSUSY 3.3.6 for $m_{t}=173.5 \mathrm{GeV}$ : (a) shows the region of successful EWSB (black), bad numerical convergence (yellow) and no EWSB (red). (b) displays the predicted value of $M_{Z} / \mathrm{GeV}$, as measured by the colour bar to the right hand side.

for a given squark and gluino mass (assuming a particular high-scale model), an uncertainty on the lightest neutralino mass which results in a theoretical uncertainty upon cut efficiencies. Currently, these sources of uncertainty are not taken into account when interpreting experimental SUSY searches in terms of particular high-scale models, and should be. However, we have shown that they are significant and so must be taken into account for any robust interpretation of data in terms of some constrained model of SUSY breaking at a high scale. The significance of the uncertainties is likely to increase as the centre of mass energy of the collider increases, since the probed region covers more of the uncertain region of parameter space. In the CMSSM in particular, such uncertainties should not have a significant impact on SUSY discovery, since the uncertainties occur in a régime of large scalar masses, so discovery is based upon the production of the other lighter sparticle states. But they do have a large impact on whether the CMSSM is a viable model or not (as opposed to some competing SUSY breaking mediation model, for instance).

We now provide recommendations for those wishing to present robust interpretations of searches for supersymmetric particles, which one should apply when considering high-scale and constrained models of SUSY breaking such as the CMSSM:

1. Generate default spectra using using a $m_{t}$ input value that is $2 \sigma$ plus a theoretical uncertainty (currently $0.8 \mathrm{GeV}$ ) higher than the central value. This ensures that one is not erroneously ruling out a region of parameter space for not breaking electroweak symmetry correctly.

2. Include a similar parametric variation of $m_{t}$ when estimating uncertainties on the interpretation of any search in the region close to the EWSB boundary, since it can 
change the kinematics of SUSY cascade chain decays, and therefore the efficiencies. It is intended that the theoretical uncertainty will be reduced in the future by including higher order effects from Refs. [32, 33] in SOFTSUSY.

3. Use a recent version of SOFTSUSY. In version 3.3.6, we have improved the numerical convergence of SOFTSUSY near the boundary of EWSB. We have also improved the diagnosis of an accurate solution of the RGEs and boundary conditions by using the difference between the value of the $Z$ mass predicted and the experimental value.

We hope that ATLAS and CMS, as well as others, will adopt these recommendations as well as the Les Houches Recommendations [39], leading to more robust interpretations of the searches.

\section{Acknowledgements}

This work has been partially supported by STFC. We thank other members of the Cambridge SUSY working group for helpful comments.

\section{References}

[1] ATLAS Collaboration Collaboration, G. Aad et. al., Search for squarks and gluinos with the ATLAS detector in final states with jets and missing transverse momentum using 4.7 $\mathrm{fb}^{-} 1$ of $\sqrt{(\mathrm{s})}=7 \mathrm{TeV}$ proton-proton collision data, 1208.0949.

[2] CMS Collaboration Collaboration, S. Chatrchyan et. al., Search for new physics in the multijet and missing transverse momentum final state in proton-proton collisions at $\sqrt{s}=7$ $\mathrm{TeV}, 1207.1898$.

[3] P. Fayet, Supersymmetry and Weak, Electromagnetic and Strong Interactions, Phys.Lett. B64 (1976) 159.

[4] P. Fayet, Spontaneously Broken Supersymmetric Theories of Weak, Electromagnetic and Strong Interactions, Phys.Lett. B69 (1977) 489.

[5] G. R. Farrar and P. Fayet, Phenomenology of the Production, Decay and Detection of New Hadronic States Associated with Supersymmetry, Phys.Lett. B76 (1978) 575.

[6] P. Fayet, Relations Between the Masses of the Superpartners of Leptons and Quarks, the Goldstino Couplings and the Neutral Currents, Phys.Lett. B84 (1979) 416.

[7] S. Dimopoulos and h. Georgi, Softly Broken Supersymmetry and SU(5), Nucl.Phys. B193 (1981) 150.

[8] B. Allanach, SOFTSUSY: a program for calculating supersymmetric spectra, Comput.Phys.Commun. 143 (2002) 305-331 [hep-ph/0104145].

[9] J. L. Feng, K. T. Matchev and D. Sanford, Focus Point Supersymmetry Redux, Phys.Rev. D85 (2012) 075007 [1112.3021].

[10] K. Matchev and R. Remington, Updated templates for the interpretation of LHC results on supersymmetry in the context of mSUGRA, 1202.6580. 
[11] K. L. Chan, U. Chattopadhyay and P. Nath, Naturalness, weak scale supersymmetry and the prospect for the observation of supersymmetry at the Tevatron and at the CERN LHC, Phys.Rev. D58 (1998) 096004 [hep-ph/9710473].

[12] J. L. Feng, K. T. Matchev and T. Moroi, Multi - TeV scalars are natural in minimal supergravity, Phys.Rev.Lett. 84 (2000) 2322-2325 [hep-ph/9908309].

[13] J. L. Feng, K. T. Matchev and T. Moroi, Focus points and naturalness in supersymmetry, Phys.Rev. D61 (2000) 075005 [hep-ph/9909334].

[14] CMS Collaboration Collaboration, S. Chatrchyan et. al., Observation of a new boson at a mass of $125 \mathrm{GeV}$ with the CMS experiment at the LHC, Phys.Lett. B716 (2012) 30-61 [1207.7235].

[15] ATLAS Collaboration Collaboration, G. Aad et. al., Observation of a new particle in the search for the Standard Model Higgs boson with the ATLAS detector at the LHC, Phys.Lett. B716 (2012) 1-29 [1207.7214].

[16] J. L. Feng and D. Sanford, A Natural $125 \mathrm{GeV}$ Higgs Boson in the MSSM from Focus Point Supersymmetry with A-Terms, Phys.Rev. D86 (2012) 055015 [1205.2372].

[17] S. Akula, P. Nath and G. Peim, Implications of the Higgs Boson Discovery for mSUGRA, Phys.Lett. B717 (2012) 188-192 [1207.1839].

[18] P. Nath, Higgs Physics and Supersymmetry, 1210.0520.

[19] S. P. Martin and M. T. Vaughn, Two loop renormalization group equations for soft supersymmetry breaking couplings, Phys.Rev. D50 (1994) 2282 [hep-ph/9311340].

[20] S. AbdusSalam, B. Allanach, H. Dreiner, J. Ellis, U. Ellwanger et. al., Benchmark Models, Planes, Lines and Points for Future SUSY Searches at the LHC, Eur.Phys.J. C71 (2011) 1835 [1109.3859].

[21] S. Abel and B. Allanach, The Quasifixed MSSM, Phys.Lett. B415 (1997) 371-382 [hep-ph/9707436].

[22] B. Allanach, J. Hetherington, M. A. Parker and B. Webber, Naturalness reach of the large hadron collider in minimal supergravity, JHEP 0008 (2000) 017 [hep-ph/0005186].

[23] V. Balasubramanian, P. Berglund, J. P. Conlon and F. Quevedo, Systematics of moduli stabilisation in Calabi-Yau flux compactifications, JHEP 0503 (2005) 007 [hep-th/0502058].

[24] A. Barr, C. Lester, M. A. Parker, B. Allanach and P. Richardson, Discovering anomaly mediated supersymmetry at the LHC, JHEP 0303 (2003) 045 [hep-ph/0208214].

[25] S. Abel, M. J. Dolan, J. Jaeckel and V. V. Khoze, Pure General Gauge Mediation for Early LHC Searches, JHEP 1012 (2010) 049 [1009.1164].

[26] T. T. Yanagida and N. Yokozaki, Focus Point in Gaugino Mediation Reconsideration of the Fine-tuning Problem , 1301.1137.

[27] Particle Data Group Collaboration, J. Beringer et. al., Review of Particle Physics (RPP), Phys.Rev. D86 (2012) 010001.

[28] B. Allanach, S. Kraml and W. Porod, Theoretical uncertainties in sparticle mass predictions from computational tools, JHEP 0303 (2003) 016 [hep-ph/0302102].

[29] B. Allanach, A. Djouadi, J. Kneur, W. Porod and P. Slavich, Precise determination of the neutral Higgs boson masses in the MSSM, JHEP 0409 (2004) 044 [hep-ph/0406166]. 
[30] A. Bednyakov, D. Kazakov and A. Sheplyakov, On the two-loop O (alpha**2(s)) corrections to the pole mass of the t-quark in the MSSM, Phys.Atom.Nucl. 71 (2008) 343-350 [hep-ph/0507139].

[31] A. Bednyakov, Some two-loop threshold corrections and three-loop renormalization group analysis of the MSSM, 1009.5455.

[32] A. Bednyakov, A. Onishchenko, V. Velizhanin and O. Veretin, Two loop O(alpha-s**2) MSSM corrections to the pole masses of heavy quarks, Eur.Phys.J. C29 (2003) 87-101 [hep-ph/0210258].

[33] P. Ferreira, I. Jack and D. Jones, The Three loop SSM beta functions, Phys.Lett. B387 (1996) 80-86 [hep-ph/9605440].

[34] ATLAS Collaboration Collaboration, G. Aad et. al., Expected Performance of the ATLAS Experiment - Detector, Trigger and Physics, 0901.0512.

[35] W. Beenakker, R. Hopker, M. Spira and P. Zerwas, Squark and gluino production at hadron colliders, Nucl.Phys. B492 (1997) 51-103 [hep-ph/9610490].

[36] P. Z. Skands, B. Allanach, H. Baer, C. Balazs, G. Belanger et. al., SUSY Les Houches accord: Interfacing SUSY spectrum calculators, decay packages, and event generators, JHEP 0407 (2004) 036 [hep-ph/0311123].

[37] T. J. LeCompte and S. P. Martin, Compressed supersymmetry after 1/fb at the Large Hadron Collider, Phys.Rev. D85 (2012) 035023 [1111.6897].

[38] Particle Data Group Collaboration, K. Nakamura et. al., Review of particle physics, 2010-2011. review of particle properties, J. Phys. G 37 (2010) 075021.

[39] S. Kraml, B. Allanach, M. Mangano, H. Prosper, S. Sekmen et. al., Searches for New Physics: Les Houches Recommendations for the Presentation of LHC Results, Eur.Phys.J. C72 (2012) 1976 [1203.2489]. 